\title{
Oportunidad de exportación de aguacate Hass a Francia
}

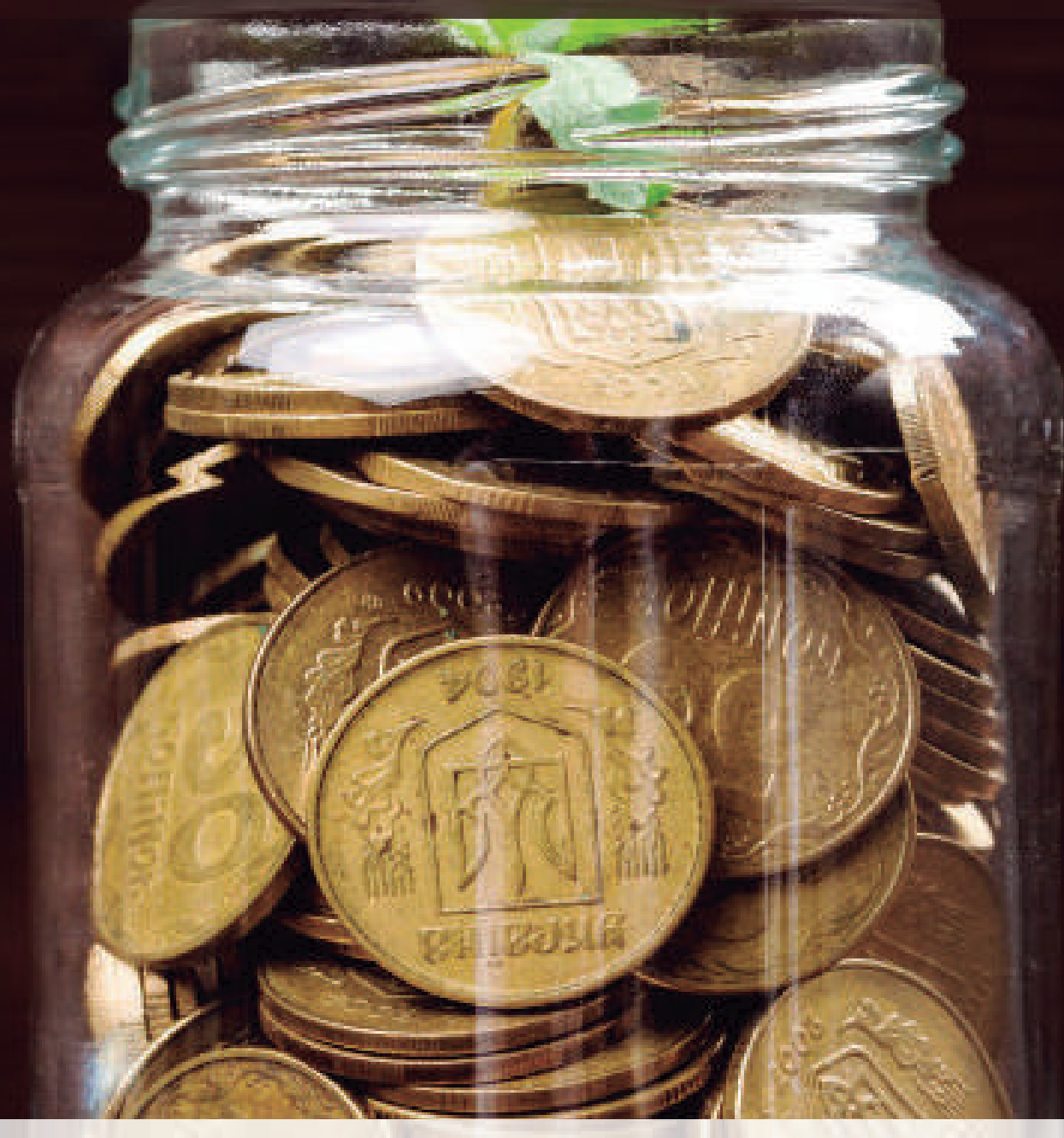

ANA ELOISA GARZÓN (ID http://orcid.org/0000-0001-6863-1894 Fundación Universitaria San Mateo / aegarzon@sanmateo.edu.co

Reconocimiento-SinObraDerivada 4.0 Internacional (CC BY-NC-ND) 
Artículo de investigación

Recepción: 13 de abril de 2020

Aceptación: 06 de agosto de 2020

Cómo citar este artículo

Garzón, A. (2020). Oportunidad de exportación de aguacate Hass a Francia. Revista Colombiana de Ciencias Administrativas 2(1), pp. 78 - 102 Recuperado a partir de: http://cipres.sanmateo.edu.co/index.php/rcca

\section{Oportunidad de exportación de aguacate Hass a Francia}

\section{Resumen:}

Las cadenas productivas en los últimos años han incentivado la internacionalización de los productos del sector agrícola del país. Con un adecuado apoyo a los productores de aguacate Hass cultivado en el departamento del Tolima, a través de emprendimientos asociativos solidarios, se pretendió buscar la manera de eliminar canales de distribución innecesarios, con el fin de que tuvieran la oportunidad de exportar directamente y así obtener una utilidad más justa. Por esa razón, desde la academia se procuró proveer a los cultivadores de toda la información, así como las herramientas requeridas para llevar sus productos a los mercados externos de manera directa, eliminando intermediarios que disminuyen la competitividad en el exterior. Así, a través de recolección y análisis de información sobre requisitos de admisibilidad, aprovechamiento de acuerdos al mercado de Francia; además, la preparación con base en la experiencia de algunas herramientas de apoyo como oferta económica, gastos logísticos y la formulación de un plan exportador para su desarrollo que les permita llegar a los mercados externos de manera directa con mayores beneficios y una ventaja competitiva más eficiente.

Palabras clave: internacionalización; cadena productiva; aguacate Hass; exportación directa; viabilidad.

\section{Hass avocado export opportunity to France}

\section{Abstract:}

In recent years, the production chains have encouraged the internationalization of the agricultural sector products from Colombia, so 
with adequate support to Hass avocado growers in Tolima's department. It was sought to find a way to eliminate channels of unnecessary distribution to have the opportunity to export directly and thus obtain a more just utility. For that reason, from the academy it was sought to provide the growers with all the information and tools that were required to carry its products to external markets directly by eliminating intermediaries that decrease competitiveness abroad, as well as through the collection and analysis of information on eligibility requirements, use of agreements to the French market and preparation based on experience of some support tools such as financial offer, logistics expenses costs and the formulation of an export plan for its development that allows them to reach external markets directly with greater benefits and a more efficient competitive advantage.

Keywords: Internationalization; production chain; Hass avocado; direct export; viability.

\section{Introducción}

Las cadenas productivas en el país han venido aportando grandes beneficios al sector agrícola; en tanto han recibido más impulso por parte del Gobierno Nacional, para no desestimar la producción de este sector como oferta exportable del país. Así, a través de los emprendimientos asociativos solidarios se ha logrado generar más oportunidades para la internacionalización de los productos, como es el caso de cultivadores de aguacate de variedad Hass, ubicado en el departamento del Tolima.

Los cultivadores se han agrupado para el cultivo y comercialización en el exterior de su producto, dando la oportunidad a la academia de apoyarlos en ese camino; así, a través de los semilleros de investigación se aportan los conocimientos en materia de procedimientos y oportunidades en los mercados externos.

Con este aporte pueden llevar a cabo la exportación de su producto en los mercados externos directamente; tienen la oportunidad de obtener no solo un precio justo por la labor, sino además eliminar intermediarios que disminuyen las utilidades al productor. Entonces, a través de la transferencia de conocimiento existen más oportunidades de negocios de manera directa; forjan mayores oportunidades como generación de empleo local, productos de mejor calidad durante todas las épocas del año y a un precio competitivo en el exterior. 


\section{Marco teórico}

Colombia es un país por excelencia agrícola, catalogado por la Organización de las Naciones Unidas para la Alimentación y la Agricultura (FAO) como la despensa agrícola del mundo; cuenta con gran variedad de oportunidades alimentarias producidas en diferentes departamentos. En cuanto al sector agroindustrial, posee variedad de frutos y hortalizas lo que se reduce en una gran oferta de productos agrícolas, suficiente para el abastecimiento local y la oportunidad de ofrecer a los mercados externos gran variedad de productos.

Sin embargo, el sector campesino del país es afectado por situaciones como el conflicto armando que ha padecido el país por más de 50 años; la proliferación de grupos terroristas o al margen de la ley; así como la delincuencia común. Dichas situaciones han propiciado escenarios asociados con la expropiación de sus tierras y el desplazamiento forzado. Las situaciones han puesto en evidencia la capacidad insuficiente de respuesta real, por parte del Estado colombiano.

No obstante, el posconflicto (con la firma del acuerdo de paz en 2016) trajo consigo nuevas oportunidades a los campesinos y cultivadores del país. Tal es el caso de los emprendimientos asociativos solidarios, aportan a la recuperación de tierras y asignación de nuevos espacios para cultivar. Brindan una mejor calidad de vida y nuevas oportunidades de prácticas modernas como el agro-sostenible.

En los sectores más vulnerables del país se empezaron a organizar las asociaciones solidarias, como es el caso de los productores de aguacate de variedad Hass. De la mano de Seven Capital Group (una entidad asociativa de productores), en alianza con la Fundación Universitaria San Mateo, se desarrolló un proyecto encaminado a proporcionar a los productores asociados herramientas para que sean partícipes activos de su propio desarrollo y así, poder enfrentar las barreras que les impide llevar sus productos de manera directa a los mercados externos.

En este sentido, eliminan a los intermediarios, tales como las comercializadoras quienes, finalmente, obtenían los mayores dividendos sobre estas operaciones. En esos términos, los agricultores eran pagados desde precios muy bajos por sus productos; en muchas ocasiones no reflejaban ni siquiera la inversión, con la finalidad de poner estos productos a un precio competitivo en los mercados internacionales. 
La presente investigación consistió en proporcionar toda la información a la asociación Seven Capital Group, teniendo como mercado objetivo Francia. Para ello, se tuvieron en cuenta requisitos de admisibilidad, oportunidad comercial de aprovechamiento, demanda, oferta, priorización de factores críticos de éxito, procesos logísticos, costos, desarrollo de oferta exportadora, oferta económica y la estructura de un plan exportador para su aplicación. Lo anterior, de acuerdo con el modelo que estableciera la asociación, propiciando que estos agricultores se conviertan en los vendedores directos.

Los departamentos más productores de aguacate en Colombia son Antioquia, Bolívar, Cauca, Cesar, Caldas, Santander, Valle del Cauca, Risaralda y Quindío, según el Ministerio de Agricultura. De tal manera, existen en el país cerca de 13.000 productores en 18.113 unidades productivas que tienen como principal actividad económica el cultivo del aguacate. El consumo per cápita por año es de 3.8 kilogramos (kg), las variedades cultivadas en Colombia corresponden a papelillo, Hass, choquette, santana, trinidad, entre los más representativos.

Ahora bien, en cuanto a hectáreas sembradas por zona, la variedad, la producción en toneladas y el porcentaje de producción frente al nivel nacional, son datos que encontramos en la Tabla 1.

Tabla 1. Áreas de producción

\begin{tabular}{|c|c|c|c|c|}
\hline ZONA & $\begin{array}{c}\text { Áreas } \\
\text { Sembrada } \\
\text { Hass } \\
\end{array}$ & Variedad & $\begin{array}{l}\text { Producción } \\
\text { (Tn) }\end{array}$ & $\begin{array}{c}\% \text { de la } \\
\text { producción } \\
\text { Nacional } \\
\end{array}$ \\
\hline $\begin{array}{c}\text { ZONA } \\
\text { OCCIDENTE } \\
\text {-EJE } \\
\text { CAFETERO } \\
\text { Antioquia, } \\
\text { Caldas, } \\
\text { Quindio, } \\
\text { Risaralda, } \\
\text { Valle del } \\
\text { Cauca } \\
\end{array}$ & 32.146 & $\begin{array}{c}\text { Hass - } \\
\text { Papelillo }\end{array}$ & 30.043 & $49 \%$ \\
\hline $\begin{array}{c}\text { ZONA } \\
\text { CENTRO } \\
\text { Tolima } \\
\end{array}$ & 15.325 & $\begin{array}{c}\text { Hass - } \\
\text { Papelillo }\end{array}$ & 71.789 & $18 \%$ \\
\hline $\begin{array}{c}\text { ZONA } \\
\text { ORIENTE } \\
\text { ( ndinamarca, } \\
\text { a, Boyacá, } \\
\text { Meta, } \\
\text { Casanare y } \\
\text { Arauca } \\
\end{array}$ & 11.420 & $\begin{array}{c}\text { Papelillo } \\
\text { verde }\end{array}$ & 24.7151 & $3 \%$ \\
\hline $\begin{array}{c}\text { ZONA } \\
\text { SANTANDER } \\
\text { Santander }\end{array}$ & 6.375 & $\begin{array}{l}\text { Criollos y } \\
\text { Antillanos }\end{array}$ & 24.7151 & $3 \%$ \\
\hline $\begin{array}{c}\text { ZONA } \\
\text { COSTA } \\
\text { ATLÁNTICA } \\
\text { Bolívar y } \\
\text { Cesar }\end{array}$ & 8.270 & $\begin{array}{l}\text { Criollos } \\
\text { Antillanos }\end{array}$ & 58.7981 & $5 \%$ \\
\hline
\end{tabular}

Fuente: Elaboración propia, a partir de Minagricultura (Agronet), 2019. 
De acuerdo con la tabla anterior, se encuentra que la zona Occidente (Eje Cafetero) es el de mayor producción de aguacate Hass y papelillo, con 32.146 hectáreas. Continúa la zona Centro donde se encuentra el Tolima con 15.325 hectáreas, con un porcentaje de la producción nacional representado en $49 \%$ y $18 \%$ respectivamente.

Tabla 2. Producción en toneladas por departamento (2017-2018)

\begin{tabular}{ccccc}
\hline DEPARTAMENTOS & $\begin{array}{c}\text { Producción } \\
(\mathbf{T n}) \mathbf{2 0 1 7}\end{array}$ & $\begin{array}{c}\text { Producción } \\
(\mathrm{Tn}) \mathbf{2 0 1 8}\end{array}$ & Participación & Variación \\
\hline TOLIMA & 5.566 & 6.016 & $22 \%$ & $8 \%$ \\
\hline QUINDÍO & 770 & 889 & $3 \%$ & $15 \%$ \\
\hline ANTIOQUIA & 8.603 & 9.482 & $34 \%$ & $10 \%$ \\
\hline CALDAS & 4.063 & 2.532 & $16 \%$ & $-38 \%$ \\
\hline CUNDINAMARCA & 6.290 & 6.940 & $25 \%$ & $10 \%$ \\
\hline SUCRE & 363 & 363 & $1 \%$ & $0 \%$ \\
\hline
\end{tabular}

Fuente: Minagricultura (Agronet), 2019.

Es importante resaltar en la Tabla 2 que en el año 2018 la producción en algunos departamentos se incrementó, teniendo en cuenta la gran demanda internacionaly la búsqueda de nuevos mercados. Esto ha permitido que cada vez se genere más producción y que la participación se incremente como en el caso del departamento de Antioquia; en el mercado esta fue del 34\% con una variación positiva del 10\%, seguido por el departamento del Tolima que llegó al 22\% de participación. Por consiguiente, contó con una variación del 8\%, a diferencia de Caldas que bajó su producción significativamente.

\section{Países productores y cultivadores.}

En cuanto a la producción de la variedad de aguacate Hass, por área cosechada el primero es México, luego Perú, Colombia, Chile e Indonesia. La participación en el mercado mundial en producción es representada en la Tabla 3, a continuación. 
Tabla 3. Producción a nivel mundial

\begin{tabular}{llll}
\hline \multicolumn{1}{c}{ Puesto } & \multicolumn{1}{c}{ País } & $\begin{array}{c}\text { Área } \\
\text { Cosechada 2017 }\end{array}$ & Participación \% \\
\hline 1 & México & 180.536 & $59 \%$ \\
\hline 2 & Perú & 37.871 & $12 \%$ \\
\hline 3 & Colombia & 35.114 & $11 \%$ \\
\hline 5 & Chile & 29.993 & $10 \%$ \\
\hline & Indonesia & 23.957 & $8 \%$ \\
\hline
\end{tabular}

Fuente: : Elaboración propia, a partir de Minagricultura, 2018 y FAO, 2017.

Como lo evidencia la Tabla 4, es importante determinar que, a nivel mundial, en producción, por tonelada para el año 2017 y la participación la marca en el primer puesto México, segundo República Dominicana, tercero Perú, encontrándose Colombia en el cuarto puesto con una participación del $11 \%$. Es relativamente muy baja, teniendo en cuenta la cantidad de hectáreas que siembran al año en los diferentes departamentos.

Tabla 4. Ranking mundial

\begin{tabular}{llll}
\hline Puesto & País & $\begin{array}{c}\text { Producción } \\
\text { Ton 2017 }\end{array}$ & $\begin{array}{l}\text { Participación } \\
\text { \% }\end{array}$ \\
\hline 1 & México & 2.021 .609 & $52 \%$ \\
\hline & República & & \\
2 & Dominicana & 643.443 & $17 \%$ \\
\hline 3 & Perú & 487.272 & $13 \%$ \\
\hline 4 & Colombia & 403.184 & $10 \%$ \\
\hline 5 & Indonesia & 326.284 & $8 \%$ \\
\hline & TOTAL & $\mathbf{3 . 8 8 1 . 7 9 2}$ & $\mathbf{1 0 0 \%}$ \\
\hline
\end{tabular}

Fuente: Elaboración propia, a partir de Minagricultura, 2018 y FAO, 2017.

\section{Indicadores de la Unión Europea.}

La Unión Europea es un grupo económico conformado por 28 países, creado luego de la Segunda Guerra Mundial, con un sistema económico basado en el euro, logrando una estabilidad económica. Cuenta con 66.954.000 millones de habitantes (2017). Según cifras del Ministerio de Comercio Industria y Turismo, es el grupo económico más grande a nivel mundial, convirtiéndose en la primera exportadora de productos manufacturados y servicios. Cuenta con más de 30 acuerdos comerciales y otros tantos en proceso de negociación; con Colombia tiene firmado desde el 2012 junto con Perú. Es un acuerdo de alcance parcial, con preferencias 
arancelarias a la mayoría del universo arancelario, sobre todo de los productos del sector agrícola, entre otros sectores.

Tabla 5. Principales países exportadores a Francia (2013-2017)

\begin{tabular}{ccccccc}
\hline & Exportadores & $\mathbf{2 0 1 3}$ & $\mathbf{2 0 1 4}$ & $\mathbf{2 0 1 5}$ & $\mathbf{2 0 1 6}$ & $\mathbf{2 0 1 7}$ \\
\hline \multirow{2}{*}{1} & \multirow{2}{*}{ Brasil } & USD & USD & USD & USD & USD \\
& \multirow{2}{*}{2.536 .907} & 3.786 .094 & 3.238 .640 & 2.980 .683 & 3.331 .208 \\
\hline \multirow{2}{*}{ México } & USD & USD & USD & USD & USD \\
& \multirow{2}{*}{ Chile } & USD & USD & USD & USD & USD \\
\multirow{2}{*}{3} & 1.453 .651 & 1.390 .417 & 1.143 .115 & 1.103 .786 & 1.231 .411 \\
\hline \multirow{2}{*}{4} & \multirow{2}{*}{ Colombia } & USD & USD & USD & USD & USD \\
& & 706.801 & 473.912 & 421.896 & 419.143 & 562.537 \\
\hline \multirow{2}{*}{5} & \multirow{2}{*}{ Perú } & USD & USD & USD & USD & USD \\
& & 405.384 & 430.931 & 477.601 & 491.992 & 517.865 \\
\hline \multirow{2}{*}{6} & \multirow{2}{*}{ Ecuador } & USD & USD & USD & USD & USD \\
& & 435.848 & 450.819 & 398.651 & 410.420 & 450.904 \\
\hline \multirow{2}{*}{7} & \multirow{2}{*}{ Argentina } & USD & USD & USD & USD & USD \\
& & 615.707 & 491.047 & 488.550 & 494.832 & 417.793 \\
\hline \multirow{2}{*}{8} & Trinidad y & USD & USD & USD & USD & USD \\
& Tobago & 315.841 & 257.607 & 221.994 & 199.952 & 296.724 \\
\hline \multirow{2}{*}{9} & \multirow{2}{*}{ Costa Rica } & USD & USD & USD & USD & USD \\
& & 269.869 & 235.332 & 254.573 & 223.336 & 252.318 \\
\hline \multirow{2}{*}{10} & República & USD & USD & USD & USD & USD \\
& Dominicana & 144.572 & 158.695 & 169.938 & 169.612 & 164.929 \\
\hline \multirow{2}{*}{} & & & & & &
\end{tabular}

Fuente: Cámara de Comercio Internacional, 2017.

La Tabla 5 muestra que, entre los mayores exportadores de productos y servicios a Francia, corresponde en su orden Brasil México y Chile. Colombia es el cuarto exportador al mercado objetivo con poca estabilidad durante los últimos cinco (5) años.

\section{Comercio desde Colombia.}

Como se mencionó anteriormente, el acuerdo firmado junto con Perú y la Unión Europea ha permitido a Colombia la entrada de nuevas oportunidades y por supuesto nuevos mercados. Aunque desde su firma en 2013 el país ha debido afrontar requerimientos estrictos de origen, trazabilidad y otras barreras no comerciales; los empresarios colombianos han realizado un trabajo dedicado y grandes esfuerzos de inversión tecnológica, técnica y financiera. Esto ha permitido acceder cada vez más fácil al mercado europeo; así como la consolidación de compañías que según Legiscomex para el año 2017 y 2018 han sido constantes en el ingreso al mercado europeo con variedad de productos nuevos, pero especialmente consolidándose cada vez con el aguacate tipo Hass. 
Tabla 6. Principales exportadores de aguacate en Colombia

\begin{tabular}{|c|c|c|c|c|}
\hline \multirow{2}{*}{ EMPRESA } & \multicolumn{2}{|c|}{2017} & \multicolumn{2}{|c|}{2018} \\
\hline & $\begin{array}{c}\text { PESO } \\
\text { EN } \\
\text { KILOS } \\
\end{array}$ & $\begin{array}{l}\text { VALOR } \\
\text { FOB } \\
\text { USD } \\
\end{array}$ & $\begin{array}{l}\text { PESO } \\
\text { EN } \\
\text { KILOS } \\
\end{array}$ & $\begin{array}{c}\text { VALOR } \\
\text { FOB } \\
\text { USD } \\
\end{array}$ \\
\hline AVOFRUIT S.A.S. & 4.999 & $\begin{array}{c}\text { USD } \\
10.386 .14 \\
9 \\
\end{array}$ & 4.333 & $\begin{array}{c}\text { USD } \\
9.981 .671 \\
\end{array}$ \\
\hline $\begin{array}{l}\text { WESTSOLE FRUIT } \\
\text { COLOMBIA S. A. S. }\end{array}$ & 4.881 & $\begin{array}{c}\text { USD } \\
9.434 .491 \\
\end{array}$ & 3.911 & $\begin{array}{c}\text { USD } \\
9.353 .457 \\
\end{array}$ \\
\hline TROPY FRUITS S.A.S. & 1.428 & $\begin{array}{c}\text { USD } \\
2.262 .699 \\
\end{array}$ & 575 & $\begin{array}{c}\text { USD } \\
1.147 .612 \\
\end{array}$ \\
\hline $\begin{array}{c}\text { PACIFIC FRUITS } \\
\text { S.A.S. } \\
\end{array}$ & 5.408 & $\begin{array}{c}\text { USD } \\
9.813 .374 \\
\end{array}$ & 2.139 & $\begin{array}{c}\text { USD } \\
3.670 .982 \\
\end{array}$ \\
\hline $\begin{array}{c}\text { ASOCIACION DE } \\
\text { PRODUCTORES DE } \\
\text { AGUACATE DE EL } \\
\text { RETIRO SAT } \\
\end{array}$ & 3.444 & $\begin{array}{c}\text { USD } \\
6.547 .651\end{array}$ & 1.641 & $\begin{array}{c}\text { USD } \\
4.113 .605\end{array}$ \\
\hline $\begin{array}{c}\text { C.I WOLF \& WOLF } \\
\text { LATINAMERICA } \\
\text { LTDA }\end{array}$ & 228 & $\begin{array}{c}\text { USD } \\
575.582\end{array}$ & 401 & $\begin{array}{c}\text { USD } \\
929.106\end{array}$ \\
\hline $\begin{array}{c}\text { EUROFRESH } \\
\text { COLOMBIA S.A.S }\end{array}$ & 30 & $\begin{array}{l}\text { USD } \\
40.757 \\
\end{array}$ & & \\
\hline OCATI S.A. 5 & 4 & $\begin{array}{c}\text { USD } \\
152.973 \\
\end{array}$ & & \\
\hline HASSPACOL S.A.S. 1 & .137 & $\begin{array}{c}\text { USD } \\
1.615 .488 \\
\end{array}$ & 614 & $\begin{array}{c}\text { USD } \\
1.248 .296 \\
\end{array}$ \\
\hline $\begin{array}{l}\text { F L P COLOMBIA } \\
\text { S.A.S. } \\
\end{array}$ & 365 & $\begin{array}{c}\text { USD } \\
626.098 \\
\end{array}$ & 501 & $\begin{array}{c}\text { USD } \\
1.050 .763 \\
\end{array}$ \\
\hline $\begin{array}{l}\text { FRUTALES LAS } \\
\text { LAJAS S. A }\end{array}$ & 801 & $\begin{array}{c}\text { USD } \\
1.365 .328\end{array}$ & 289 & $\begin{array}{c}\text { USD } \\
562.030\end{array}$ \\
\hline $\begin{array}{c}\text { JARDIN EXOTICS } \\
\text { S.A.S. } \\
\end{array}$ & 669 & $\begin{array}{c}\text { USD } \\
1.499 .627 \\
\end{array}$ & 1.611 & $\begin{array}{c}\text { USD } \\
3.456 .063 \\
\end{array}$ \\
\hline $\begin{array}{c}\text { HATO LA MACANA } \\
\text { SAS } \\
\end{array}$ & 364 & $\begin{array}{c}\text { USD } \\
839.086 \\
\end{array}$ & 66 & $\begin{array}{c}\text { USD } \\
125.969 \\
\end{array}$ \\
\hline GREEN WEST S. A. S. 1 & .650 & $\begin{array}{c}\text { USD } \\
2.417 .855\end{array}$ & 1.007 & $\begin{array}{c}\text { USD } \\
1.926 .762\end{array}$ \\
\hline
\end{tabular}

Fuente: Elaboración propia, a partir de Legiscomex, 2018.

\section{Condiciones de acceso exigidas para la admisibilidad del aguacate Hass.}

El Instituto Agropecuario Colombia es el encargado de determinar las condiciones de manejo a los productores de aguacate en el país; así como llevar el control de estos y que se cumplan no solo con la normatividad local, sino que además pueda ser aceptado en los mercados internacionales. Para este caso exige que se certifiquen lo que se denomina huerto exportador.

De igual manera el predio también tiene un proceso de certificación; adicionalmente, la planta empacadora del mismo también debe contar con esta condición para que el producto pueda ser exportado. Finalmente, 
certificaciones adicionales de procesos de calidad como el caso del GLOBALG.A.P, una certificación de buenas prácticas agrícolas. Estas normas ayudan a los productores a cumplir los criterios de toda Europa respecto a la inocuidad alimentaria, métodos de producción sostenible, bienestar de los trabajadores y de los animales, y el uso responsable del agua. Para los productores significa un mayor ahorro, evita largos procesos de auditorías, asimismo reduce desperdicio de recursos vitales (GLOBALG.A.P, 2018). Por lo anterior, los requisitos específicos de origen son:

- Certificación de buenas prácticas de manufactura (planta empacadora).

- Certificación de buenas prácticas agrícolas (productor).

- Certificación de ICA huerto exportador.

- Certificación ICA exportadora de vegetales frescos.

- Certificación Planta empacadora "Packing House".

- Certificación Global Gap (opcional).

\section{Logística, distribución física internacional.}

Es el proceso logístico que consiste en situar un producto en los mercados externos, cumpliendo los términos negociados entre vendedor y comprador. Su principal objetivo es la reducción al máximo de los tiempos, los costos y los riesgos que la operación pueda generar durante el trayecto desde el punto de salida u origen hasta punto de llegada o destino.

\section{Clasificación arancelaria.}

El nombre técnico del aguacate es Persea americana; su nombre comercial corresponde a aguacate o palta, nombre proveniente del lenguaje quechua. Es utilizado para referirse a una fruta, variedad Hass. En el sistema armonizado se encuentra clasificado con la partida arancelaria 08.04.40.00.00

\section{Requisitos específicos de producto para la Unión Europea.}

Según información de la entidad agrícola encarga del control de importación de productos del sector agrícola, se encuentran los siguientes requisitos específicos y generales (Comisión Europea, s.f):

- Control de contaminantes en alimentos. 
- Control de residuos de plaguicidas en alimentos de origen vegetal y animal.

- Control sanitario de alimentos de origen no animal.

- Control sanitario de alimentos genéticamente modificados y nuevos alimentos.

- Controles fitosanitarios.

- Trazabilidad, cumplimiento y responsabilidad en alimentos y piensos.

- Etiquetado de alimentos.

- Normas de comercialización de hortalizas y frutas frescas (solo aplicable a fruta fresca).

- Productos de producción ecológica (voluntario).

Todos los requisitos anteriormente descritos son el equivalente en Colombia al registro exportador del Instituto Colombiano Agropecuario (ICA) (huerto exportador), registro de planta empacadora y registro exportador de productos frescos para producto provenientes de terceros países los cuales cuentan con una tarifa estándar de impuesto sobre el valor añadido (IVA) de 5.5\%. No obstante, cuando es proveniente de Colombia, que cuenta con acuerdo de libre comercio en compañía de Perú, no paga derechos e impuestos a las importaciones ni tampoco tarifa de IVA.

- Empaque y embalaje: Cajas de cartón corrugado plegadiza apta para furas y hortaliza de acuerdo con la normatividad europea para el manejo de la carga:

- Dimensión de cajas: $35.5 \mathrm{~cm}$ largo por $28.5 \mathrm{~cm}$ ancho y $10 \mathrm{~cm}$ de alto.

- Calibres: 12 a 20 con alveolo cada caja.

- Peso por caja: $4 \mathrm{~kg}$.

- Embalaje: Estiba de madera certificada euro pallet con medidas de $1.20 \mathrm{~m}$ de largo por $0,80 \mathrm{~cm}$ de ancho.

- Paletizado: cuatro (4) cajas acomodadas a lo largo por dos (2) cajas acomodadas a lo ancho para un total de ocho (8) cajas de base, apilamiento de máximo 20 cajas para un total de cajas por estiba de 160.

- Cajas por estiba: 160 cajas.

- Peso Pallet: $660 \mathrm{~kg}$.

- Alto pallet: $2.15 \mathrm{~m}$.

Adicionalmente se utilizan perfil de cartón o esquineros asegurados con zuncho como refuerzo estructural para el pallet. Estos refuerzos ayudarán a que las cajas queden sobre la estiba para evitar posibles daños de la mercancía. Además, evita el movimiento y daños de las cajas durante su manipulación en el transporte. 
Por la naturaleza de la carga perecedera se utiliza un contenedor de 40 pies refrigerado, de tres (3) a cinco (5) ${ }^{\circ} \mathrm{C}$, con sistema de atmosfera controlada.

\section{Perfil logístico y acceso marítimo Francia.}

Figura 1. Ruta marítima

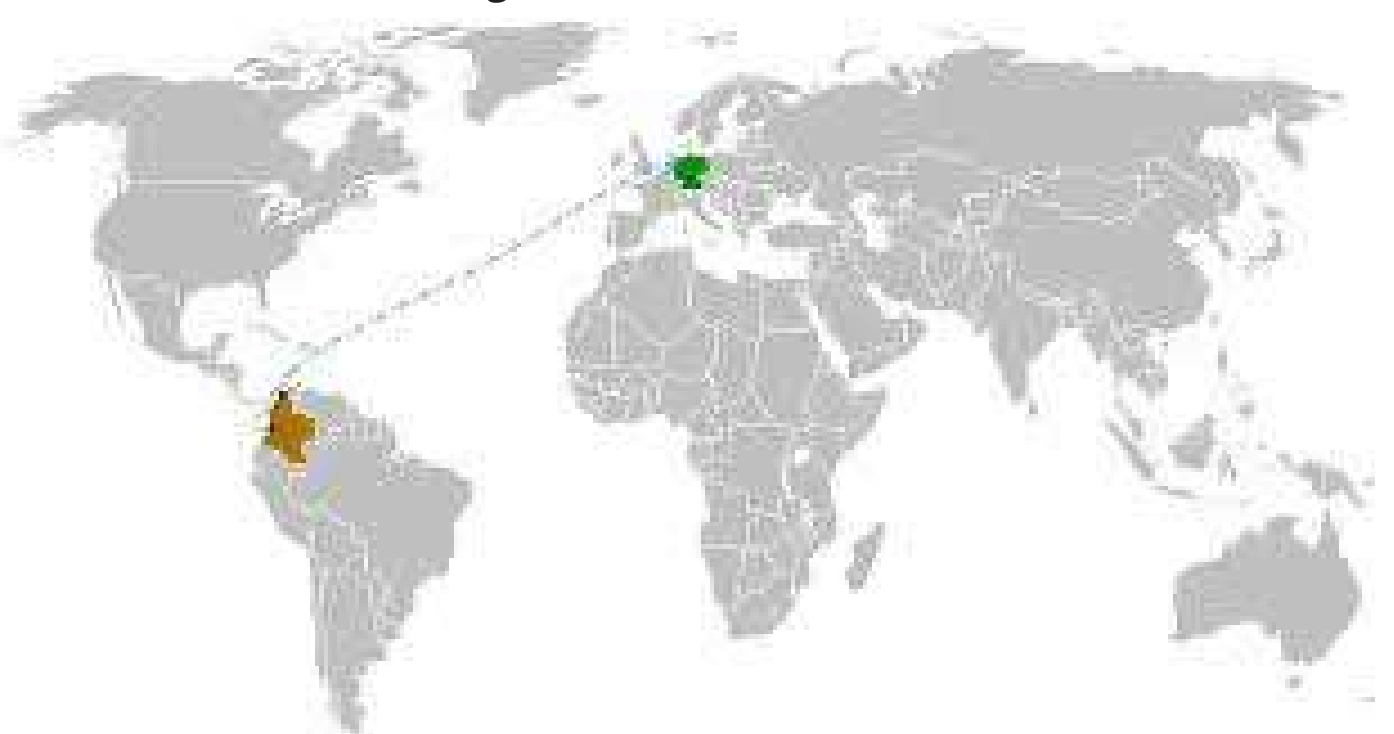

Fuente: Procolombia, 2018.

Según el Banco mundial, Francia ocupa en el mundo el puesto número 16 en lo que se refiere a su desempeño logístico para el año 2019. En cuanto a la movilización de mercancías por vía marítima, Francia cuenta con $6.000 \mathrm{~km}$ de vías fluviales son navegables; además, con una moderna infraestructura en puertos compuesto por 100 puertos entre principales y auxiliares. Entre los más comerciales encontramos a Le Havre, Marsella, Dunkirk y Brest.

El puerto de Marsella es el más grande de Francia y el tercero a nivel europeo, tiene un manejo por año aproximado de 100 millones de toneladas. Posee importantes conexiones para carreteras, viras férreas y fluviales; vive en constante desarrollo tecnológico y de infraestructura para recibir cualquier tipo de tráfico y contenedores (Procolombia, 2018). 


\section{Transporte por carretera.}

La distribución de las principales vías se encuentra organizadas de la siguiente manera de acuerdo con el ministerio de cohesión territorial de Francia, entidad encargada del control (Viajar a Francia, 2018; France Diplomatie, 2018):

Figura 2. Territorio francés

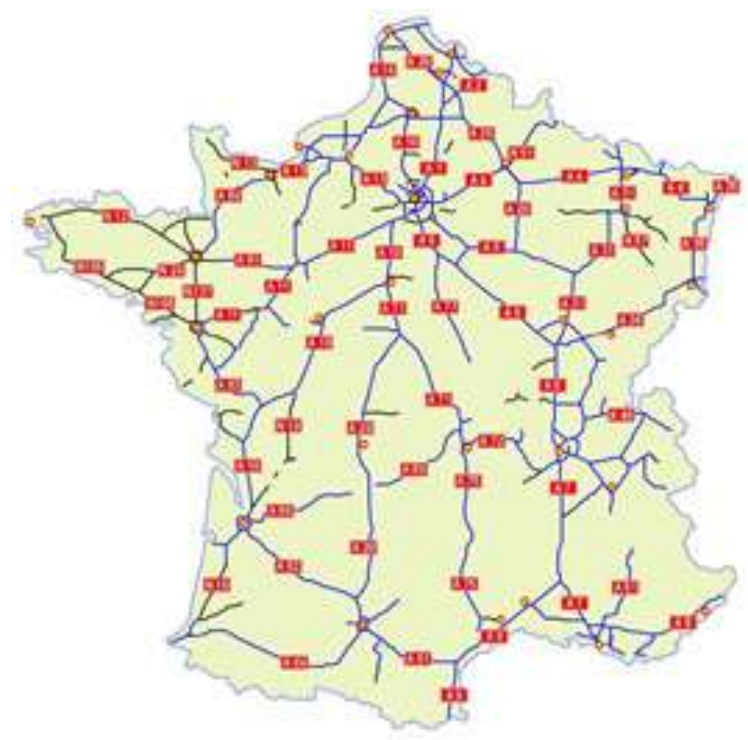

Fuente: Viajar a Francia, 2018.

\section{Cadena de Frío.}

La cadena de frío es un proceso logístico desde producción, almacenaje, distribución, embalajes, transporte, carga y descarga. Maneja una temperatura y humedad relativa controlada, durante todo el tiempo del proceso, desde el mismo momento de inicio de la producción hasta el consumidor final. Su objetivo es preservar el producto de temperaturas críticas de riesgo, evitando al máximo la proliferación bacteriana que le permita afectar la salud del consumidor final.

La implementación eficiente de actividades logísticas a lo largo de la cadena de abastecimiento es de vital importancia para garantizar las adecuadas temperaturas que requieren determinados productos perecederos hasta su disposición final. 
Para el caso del aguacate, necesita de un grado de refrigeración de tres (3) a cinco $(5)^{\circ} \mathrm{C}$, después de salir de la planta de empaque y embalaje, durante todo el proceso logístico, sin que esta cadena se rompa. Lo anterior, puesto que puede afectar las características físicas del producto y por ende su grado de maduración.

Para su transporte por carretera se utiliza un vehículo refrigerado el cual permite reducir la temperatura del interior de la unidad de transporte o contenedor a $-20^{\circ} \mathrm{C}$ y de mantenerla, inclusive, para una temperatura ambiental exterior media de $30^{\circ} \mathrm{C}$. Lo anterior, de acuerdo con las normas establecidas para el transporte por carretera del Ministerio de Transporte. El furgón refrigerado son los semirremolques más eficientes; cuenta con mayor capacidad de carga y un peso vehicular más bajo.

\section{Sistema de atmosfera controlada.}

El sistema de atmósfera controlada se utiliza para retardar la maduración del producto durante el proceso logístico y que llegue en óptimas condiciones fisicoquímica. Este sistema permite seleccionar la composición de los gases al interior del contenedor, así que el usuario puede escoger la cantidad de Dióxido de Carbono (CO2) y Oxígeno (O2) que necesite para el producto y el resultante será ocupado en la mayor parte por el Nitrógeno (N2) y un mínimo porcentaje de otros gases inertes. Este sistema permite ser adaptado a cualquier contenedor refrigerado, sin modificar de ninguna forma su estructura o maquinaria y convertirlo en un contenedor de atmósfera controlada exclusivo para un solo viaje.

\section{Incoterms 2010.}

Son términos de negociación internacional, establecidos por la Cámara de Comercio Internacional que determinan las condiciones tanto de comprador como vendedor en una negociación dentro del contrato de compraventa. Estos identifican la trasferencia de la propiedad de las mercancías, los riesgos, lugar acordado de entrega de las mercancías, las responsabilidades de contratación y pago del transporte, el seguro y la entrega de los documentos respectivos.

Para la investigación se trabajó con los incoterms EXW, FOB Y CIF, para determinar las responsabilidades tanto de vendedor como de comprador en la operación que se pretende llevar a cabo, así como los costos. 
Tabla 10. Incoterms

\begin{tabular}{|c|c|c|c|c|c|}
\hline INCOTERMS & SIGNIFICADO & RESPONSABILIDADES VENDEDOR & & RESPONSABILIDADES C & COMPRADOR \\
\hline $\begin{array}{c}\text { EXW } \\
\text { Ex Works } \\
\text { Multimodal }\end{array}$ & $\begin{array}{l}\text { En fábrica o lugar } \\
\text { designado }\end{array}$ & $\begin{array}{ll}\text { Entrega } \\
\text { transferencia } \\
\text { la mercancía }\end{array}$ & $\begin{array}{r}\mathrm{y} \\
\mathrm{de} \\
\mathrm{y} \\
\mathrm{de}\end{array}$ & $\begin{array}{l}\text { Pransporte interior en destino } \\
\text { Pago de impuestos }\end{array}$ & $\begin{array}{l}\text { Pago de la } \\
\text { mercancía } \\
\text { Transporte local } \\
\text { origen } \\
\text { Despacho en } \\
\text { aduana de origen } \\
\text { Gastos de salida } \\
\text { Flete internacional } \\
\text { Seguro } \\
\text { Aduana de destino }\end{array}$ \\
\hline $\begin{array}{c}\text { FOB } \\
\text { Free on Board } \\
\text { Marítimo }\end{array}$ & $\begin{array}{l}\text { El vendedor entrega la } \\
\text { mercancía al comprador } \\
\text { a bordo del medio de } \\
\text { transporte elegido }\end{array}$ & $\begin{array}{ll}- & \begin{array}{l}\text { Entrega de } \\
\text { mercancía }\end{array} \\
\text { Empaque } \\
\text { embalaje } \\
\text { Transporte } \\
\text { interno } \\
\text { origen } \\
\text { Despacho } \\
\text { aduana } \\
\text { origen } \\
\text { Gastos de salid } \\
\text { Documentos } \\
\text { Entrega de } \\
\text { mercancía }\end{array}$ & $\begin{array}{l}\text { la } \\
\text { y } \\
\text { en } \\
\text { de } \\
\text { en } \\
\text { ida } \\
\text { la }\end{array}$ & 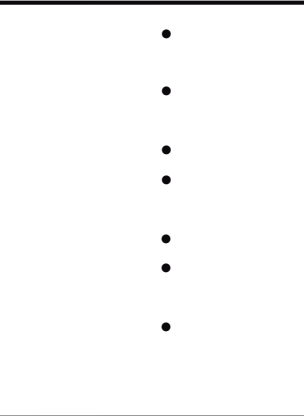 & $\begin{array}{l}\text { Pago de la } \\
\text { mercancía } \\
\text { Transporte interior } \\
\text { origen } \\
\text { Flete internacional } \\
\text { Seguros gastos en } \\
\text { destino } \\
\text { Aduana de destino } \\
\text { Transporte interior } \\
\text { en destino } \\
\text { Pago de impuestos }\end{array}$ \\
\hline $\begin{array}{c}\text { CIF } \\
\text { Cost, ins urance and } \\
\text { freigth } \\
\text { Marítimo }\end{array}$ & $\begin{array}{l}\text { Entrega en puerto de } \\
\text { destino acordado, pago } \\
\text { de flete intencional y } \\
\text { seguro }\end{array}$ & 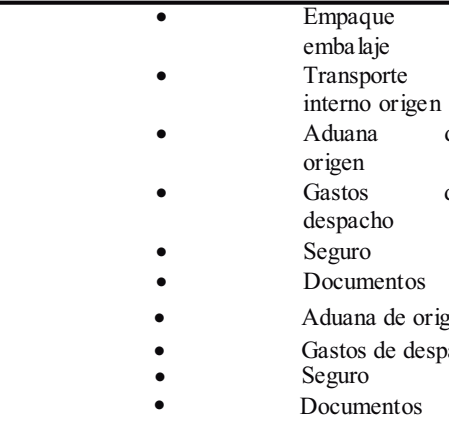 & $\begin{array}{l}\text { y } \\
\text { de } \\
\text { de } \\
\text { igen } \\
\text { pacho }\end{array}$ & $\begin{array}{l} \\
\\
\bullet \\
\bullet \\
\bullet \\
\bullet\end{array}$ & $\begin{array}{l}\text { Pago de la } \\
\text { mercancía } \\
\text { Gastos de llegada } \\
\text { Aduana destino } \\
\text { Transporte interno } \\
\text { destino } \\
\text { Impuestos }\end{array}$ \\
\hline
\end{tabular}

Fuente: Icotainers, 2018.

\section{Seguro.}

El seguro de mercancías comprende poner en las manos de una aseguradora el riesgo que las mercancías pueden sufrir durante el proceso logístico de las mismas hasta llegar a su destino final, dependiendo del término de negociación pactado entre vendedor y comprador.

\section{Canales de distribución.}

Es el medio por el cual cada empresa escoge para hacer llegar sus productos al consumidor final de la forma más eficiente, eficaz y económica posible. En el mercado en la actualidad se identifican cuatro (4) canales de 
distribución que apoyan la comercialización de los productores; quienes en sus inicios no cuentan con presencia en los mercados internacionales que puedan asumir el rol de vendedor directo de sus productos.

Por otro lado, como características generales encontramos que estos intermediarios encarecen el producto final al consumidor, por lo que se aconseja la eliminación en lo posible de estos intermediarios; es específicamente al comercializador que se pretende eliminar de la cadena.

\section{Costos de la distribución física internacional.}

Otro de los factores más importantes del proceso correspondiente al costeo, de acuerdo con el término de negociación establecido entre el vendedor y comprador. Para esta investigación se plantearon dos (2) términos FOB y CIF, sin embargo, en esta fase se consideró la posibilidad de comercializar menos producto, dada la naturaleza de la mercancía.

Igualmente, se consideró la posibilidad del envío de muestras sin valor comercial, lo que implica la utilización de un medio de transporte aéreo. Por lo tanto, se planteó el manejo de la mercancía por tres (3) agentes de carga con mayor volumen de operaciones. Además, se identificaron los costos para este medio de transporte, con incoterms equivalentes a FCA y CPT.

\section{Costos marítimos.}

Los valores se encuentran actualizados a 2019 y se estableció tasa representativa del mercado del 29 de mayo del 2019 a \$3.362,48 por dólar. En esta oportunidad para un contenedor refrigerado, con 20 euro pallet y 3.200 cajas de aguacate de diversos calibres el valor CIF, fue de USD\$25.31 por $\mathrm{kg}$, y valor EXW de USD\$1,61 por kg de aguacate.

Es de tener en cuenta que para este caso hay dos (2) factores que representan el incremento de costos para esta operación. Por un lado, corresponde el sistema de atmósfera controlada que cuenta con un elevado costo por el tiempo de trayecto y la necesidad de uso. Por otro lado, el incremento representativo del dólar, que para la primera fase se estableció sobre \$3.174,11 por dólar (Anexo 3). 


\section{Costos aéreos.}

Dada la cantidad de producto que en marítimo representa, en esta fase y teniendo en cuenta que para iniciar negociación muy seguramente la compra de producto no podría ser de este volumen, se determinaron costos vía aérea, por unidad mínima. Lo anterior correspondería a un pallet con 160 cajas y un peso de 660kg por pallet, determinando también esta cantidad con la posibilidad de envío de muestras sin valor comercial. Para este caso no se hace necesario la utilización de atmósfera controlada; el trayecto es de máximo tres (3) días sino es directo. Sin embargo, requiere el grado refrigeración de tres (3) a cinco $(5)^{\circ} \mathrm{C}$ para la inocuidad del producto.

Para este caso, se cotizaron tres (3) agentes de carga con posibilidad de oferta del flete que manejan grandes volúmenes por lo que sus tarifas pueden ser competitivas. También, teniendo el dólar a \$3.362,48, se estableció que para UPS el valor por kilo es de USD\$17,41, para DHL es de USD\$19,14 por kg y para Air France directamente un valor de USD\$18,66. Aunque en transporte aéreo el flete es más costoso, es posible llevar menor cantidad en menor tiempo (Anexo 4).

\section{Oferta exportadora.}

Teniendo en cuenta que los productores tienen total desconocimiento de los procedimientos para poder acceder a los mercados internacionales, una de las herramientas más importante es la oferta exportadora. Así, se preparó un modelo de oferta que puede apoyar el proceso de negociación con el comprador, junto con el brochure de la entidad que pueda dar a conocer más de los productores. Además, una ficha técnica del producto que le permite conocer a profundidad un poco más del producto, sus condiciones, usos, empaques, embalajes, y demás condiciones importantes para acceder a los mercados externos.

Se estableció un modelo estándar que le permita a cualquier productor gestionarlo de forma fácil. La propuesta económica es práctica al cliente, basada en las características de la investigación, en este caso, específicamente para el aguacate Hass (Anexo 1). 


\section{Oferta económica.}

Teniendo en cuenta la oferta, como una de las partes más delicada del proceso; es importante mencionar varios factores en cuanto a la estructura de costos se refiere, especialmente, la fluctuación del dólar que es un factor determinante en Colombia, debido a la volatilidad que esta presenta continuamente. En este sentido, se identifica valor por unidad, por caja y por pallet, haciendo referencia que esta puede variar dependiendo del tipo de solicitud o requerimiento del cliente (Anexo 2).

\section{Plan exportador.}

Con el fin de apoyar los procesos empresariales hacia la internacionalización de las empresas, se ha generado una estructura de un plan de exportación general. Lo anterior, con el fin de que las empresas puedan identificar cuáles son los aspectos a tener en cuenta para llegar a los mercados externos. El primer paso es identificar algunos aspectos relevantes que se deben tener en cuenta como organización. Por esta razón se presenta una guía, que surgió como una síntesis, después de consultar varios modelos dispuestos en entidades gubernamentales como Procolombia, Cámara de Comercio de Bogotá y Cámara de Comercio Internacional, de lo que debería adaptarse en las empresas para empezar. Es de anotar que el contenido, en sí, corresponderá a la estructura empresarial que se desarrolle, a su objeto social, así como a los objetivos planteados para la internacionalización.

Según Dayanna Grijalva, en su tesis de maestría identifica la importancia del plan exportador como "una herramienta muy importante para una compañía que desea comenzar a posicionarse en el exterior, este plan tiene como objetivo respuesta a una gama de preguntas que se realizan en un plan estratégico con miras a la internacionalización" (Grijalva, 2018, pp. 22).

Teniendo en cuenta la importancia para lo empresario de contar con una guía, se genera una estructura general de donde deben desprenderse. Asimismo, requerimientos adicionales que puedan surgir del objeto social, del tipo de producto, del tipo de negociación, que sería una base para el desarrollo de un plan de internacionalización de una organización (Anexo 3). 


\section{Método y diseño}

La realización de esta investigación es de tipo mixto, a través de la realización en primera fase de recolección de la información de tipo cualitativo de fuentes primarias y secundarias. Posteriormente, se organizó y realizó el análisis de la información por el método cuantitativo, aportando al final una descripción de los requisitos y procesos para llevar a cabo el proyecto.

\section{Participantes.}

La población participe de la investigación, se tomó teniendo en cuenta que Seven Capital Group está asociada con productores del departamento del Tolima. Entonces se analizó para el caso las cantidades de producción del departamento y se comparó con otros departamentos.

\section{Procedimiento.}

La investigación se desarrolló en dos (2) fases, cada una de cuatro (4) meses. La primera se enfocó en la caracterización del producto, la demanda del producto, identificación y priorización de factores críticos, identificación de normas de origen y admisibilidad y proceso logístico para el mercado objetivo.

En cuanto a la segunda fase, se elaboraron las herramientas para el producto, se analizaron costos por dos (2) medios de transportes. Una vez compilada toda la información y las herramientas (como oferta exportadora y económica, estructura del plan exportador) se entregaron a la asociación Capital Seven Group para su desarrollo.

\section{Resultados}

El mercado del aguacate tiene un gran potencial de comercialización en los mercados externos, con un aumento representativo los últimos dos (2) años. Sin embargo, el desconocimiento por parte de los productores del fruto, de las normas y requisitos para internacionalizar su producto es una gran barrera; también la falta de acompañamiento del Gobierno a los cultivadores en sus propios terrenos especialmente entidades de apoyo a las exportaciones. Además, la falta de capital de trabajo para el desarrollo de los requisitos del ICA; si bien esta entidad si presta ayuda técnica, es apenas una parte del proceso de internacionalización de los productores. 
El desconocimiento del proceso de exportación genera incertidumbre y miedo a los cultivadores. Esta es la razón para vender su producto a comercializadores que logran pagar bajos precios a los cultivadores por su producto; requieren llegar al mercado externo con precios competitivos, que son finalmente exportadores directos.

\section{Discusión de resultados.}

Durante el proceso de la investigación no se encontraron estudios previos del tema, solo análisis cuantitativos generales del sector por departamentos y países que se tomaron como base para obtener resultados actualizados (inclusive a 2019). Analizar el comportamiento, si bien es un mercado relativamente nuevo, se pudo establecer que es muy potencial, no solo para el mercado objetivo como se pensó en un principio. Adicionalmente, durante el proceso se identificaron algunos otros de relevancia, para los productores.

Asimismo, a pesar de la falta de presencia del Estado en este tipo de encadenamientos, los mismos productores han decidido organizarse y así poder llevar a cabo un proyecto que les permita llegar a los mercados externos de manera directa con sus productos, pudiendo así obtener mejores ganancias a los procesos y la motivación de seguir cultivando. Esto genera una mejor calidad de vida para todas sus familias.

\section{Conclusiones}

Con base en los resultados enunciado anteriormente encontramos que es perfectamente viable que los productores exporten de manera directa sus productos. Ha sido de vital importancia el emprendimiento asociativo solidario para dar impulso hacia una ventaja competitiva; sin embargo, requieren más apoyo técnico y financiero para el desarrollo de proyectos directos de internacionalización de este producto.

En este sentido, es posible beneficiarse del acuerdo firmado con el bloque económico de la Unión Europea, pudiendo llegar a este y otros mercados con precios competitivos y un producto de calidad. Así, la asociación de cadenas productivas les permite a estos productores lograr la internacionalización de una manera más eficiente y en bloque pudiendo beneficiar a todos y aportar al mejoramiento del agro colombiano y la calidad de vida de sus cultivadores. 


\section{Referencias}

Camara de Comercio Internacional . (2017). CCl. Obtenido de https:// www.trademap.org/Index.aspx

Comisión Europea. (s.f). De Colombia a Francia. Obtenido de http://trade.ec.europa.eu/tradehelp/es/ myexport\#?product=0804400000\&partner $=$ CO\&reporter $=F R \& t a b=a l l$

FAO . (2017). Organización de las Naciones Unidas para la Alimentación y la Agricultura. Obtenido de http://www.fao.org/faostat/es/\#country/44

France Diplomatie. (2018). Reforma del Estado y gobernanza territorial. Obtenido de https://www.diplomatie.gouv.fr/es/asuntos-globales/ gobernanza/reforma-del-estado-y-gobernanza-territorial/

Global. G.A.P. (2018). La norma mundial para las Buenas Prácticas Agrícolas. Obtenido de https://www.globalgap.org/es

Icotainers. (2018). Icoterms. Obtenido de https://www.icontainers.com/ es/incoterms/incoterm

Legiscomex. (2018). Legiscomex. Obtenido de https://www.legiscomex. com/?SSO-LC-71

Ministerio de Agricultura . (2019). Ministerio de Agricultura. Obtenido de https://www.agronet.gov.co/Paginas/inicio.aspx

Ochoa, D. D. (2018). Plan de exportación y producción orgánica para el Mercado. 22-23.

Procolombia. (2018). Perfil logístico de exportación a Francia. Obtenido de http://www.colombiatrade.com.co/herramientas/perfiles-logisticos-deexportacion-por-pais/perfil-logistico-de-exportacion-francia

Viajarafrancia.com. (2018). Carreteras en francia. Obtenido de https:// www.viajarafrancia.com/carreteras-en-francia/ 
Anexo 1. Oferta exportadora

\begin{tabular}{|c|c|}
\hline \multicolumn{2}{|c|}{ OFERTA EXPORTADORA } \\
\hline \multicolumn{2}{|c|}{$\begin{array}{l}\text { PRODUCTO: } \\
\text { El Aguacate Hass, se conoce también como "la fruta del corazón", no sólo por su similitud con el órgano vital } \\
\text { sino porque sus aceites son benéficos para reducir el colesterol y proteger el sistema cardio-circulatorio. Es la } \\
\text { variedad más conocida y comercializada. Al principio es verde; maduro y apto ya para el consumo es de color } \\
\text { violeta muy oscuro, casi negro. De piel dura, gruesa y áspera, su pulpa es amarilla y su sabor similar al de los } \\
\text { frutos secos. Es una variedad relativamente más pequeffa que el resto de las variedades que se comercializan, } \\
\text { pero con más proporción de pulpa. Tolima cuenta para el año } 2018 \text { con } 15.325 \text { hectáreas de cultivo de este } \\
\text { fruto con } 71.789 \text { toneladas de producción. }\end{array}$} \\
\hline \multicolumn{2}{|c|}{$\begin{array}{l}\text { ACUERDO COMERCIAL: } \\
\text { Nuestro mercado objetivo es la unión Europea bloque económico con el que Colombia tiene un acuerdo } \\
\text { comercial junto con Perú desde el año } 2013 \text {, con preferencia de ingreso a mercado de tipo arancelario con el } \\
\text { cumplimiento de las normas de origen establecidas por este bloque para productos provenientes del pais. } \\
\text { Como requisitos mínimos: } \\
\text { 1. Certificación buenas prácticas de manufactura. } \\
\text { 2. Certificación Buenas prácticas agrícolas. } \\
\text { 3. Certificación ICA huerto exportador. } \\
\text { 4. Certificación ICA para predio. } \\
\text { 5. Certificación Planta empacadora. }\end{array}$} \\
\hline \multicolumn{2}{|c|}{$\begin{array}{l}\text { EMPAQUE Y EMBALAJE } \\
\text { De acuerdo a la Unión Europea el empaque y el embalaje este debe estar sujeta a la legislación y normatividad } \\
\text { sobre protección al consumidor, cuidado al medio ambiente, prácticas comerciales y normas internacionales } \\
\text { establecidas para este tipo de producto. }\end{array}$} \\
\hline $\begin{array}{l}\text { EMPAQUE: } \\
\text { El aguacate se clasifica de acuerdo a la Norma Codex } \\
\text { por calibres: }\end{array}$ & $\begin{array}{l}\text { establecidas según la norma de la Unión } \\
\text { n CF1 y CF2. }\end{array}$ \\
\hline $\begin{array}{l}\text { Por el peso del fruto y por número de frutas en el } \\
\text { envase (conteo): La planta empacadora certificada } \\
\text { realiza la selección de proceso, se separa por calibres } \\
\text { y se empaca de acuerdo a la norma europea del peso } \\
\text { del fruto más pequeño no deberá ser menor al } 75 \% \\
\text { del peso del fruto más grade en el mismo envase. }\end{array}$ & $\begin{array}{l}\text { Cajas por pallet: } 160 \\
\text { Peso Pallet: } 660 \text { Kilos } \\
\text { Alto pallet: } 2.15 \text { Metros }\end{array}$ \\
\hline $\begin{array}{l}\text { Se utilizan cajas de cartón corrugado de do } \\
\text { resistente a la humedad, base modular auto } \\
\text { bajo la norma internacional para em }\end{array}$ & $\begin{array}{l}200 \text { cajas, para un peso total de carga de } 1.860 \text { Kilos } \\
\text { roximadamente }\end{array}$ \\
\hline
\end{tabular}

Fuente: Elaboración propia.

Anexo 2. Oferta económica

\begin{tabular}{|c|c|c|c|c|c|}
\hline \multicolumn{9}{|c|}{ OFERTA ECONOMICA } \\
\hline $\begin{array}{c}\text { PRODUCT } \\
\text { O }\end{array}$ & $\begin{array}{c}\text { CANTIDAD } \\
\text { AGUACATE }\end{array}$ & $\begin{array}{c}\text { PRECIO } \\
\text { UNITARIO }\end{array}$ & $\begin{array}{c}\text { PRECIO } \\
\text { TOTAL USD }\end{array}$ & TRM & $\begin{array}{c}\text { VALIDEZ DE } \\
\text { LA OFERTA }\end{array}$ \\
\hline UNIDAD & & & & $\begin{array}{c}\text { Esta oferta } \\
\text { tiene una } \\
\text { validez en } 10 \\
\text { dias. }\end{array}$ \\
\hline CAJA & & & & $\begin{array}{c}\text { Esta oferta } \\
\text { tiene una } \\
\text { validez en } 10 \\
\text { dias }\end{array}$ \\
\hline PALLET & & & & $\begin{array}{c}\text { Esta oferta } \\
\text { tiene una } \\
\text { validez en } 10 \\
\text { dias }\end{array}$ \\
\hline
\end{tabular}

Fuente: Elaboración propia. 
Anexo 3. Plan Exportador

\begin{tabular}{|l|l|}
\hline RAZON SOCIAL & NIT \\
\hline Objeto Social de la Empresa: \\
\hline Misión: \\
\hline Visión: \\
\hline Objetivos: \\
\hline Valores: \\
\hline
\end{tabular}

\section{SITUACIÓN DE LA EMPRESA}

Antecedentes perfil histórico.

Estructura organizacional.

Formalidad de empresa.

Situación económica de la empresa.

Mercadeo

Matriz DAFO

PRODUCTO

Ficha técnica (Nombre comercial, descripción general, composición, unidad de medida, usos, presentaciones)

\begin{tabular}{|l|}
\hline Calidad. \\
\hline Catalogo. \\
\hline Clasificación arancelaria. \\
\hline Volumen de producción. \\
\hline Costo de producción. \\
\hline Normas técnicas utilizadas para la fabricación. \\
\hline Requerimientos de salida del producto. \\
\hline Empaque. \\
\hline Embalaje. \\
\hline Precio de venta local. \\
\hline Términos de negociación. \\
\hline Certificados requeridos. \\
\hline
\end{tabular}

Fuente: Elaboración Propia 
Anexo 4 Costos Marítimos

\begin{tabular}{|c|c|c|c|c|c|c|c|}
\hline \multicolumn{3}{|c|}{ DESCRIPCION } & \multicolumn{2}{|c|}{ VALOR } & & & \\
\hline \multirow{7}{*}{$\begin{array}{l}\text { INFORMACION BASICA DEL } \\
\text { PRODUCTO }\end{array}$} & PRODUCTO & Aguacate Hass & & & & & \\
\hline & PARTIDA ARANCELARIA & 08.04 .40 .00 .00 & & & & & \\
\hline & UNIDAD DE VENTA & KG & & & & & \\
\hline & EMPAQUE & Caja de carton doble pared & & & & & \\
\hline & EMBALAJE & $\begin{array}{l}\text { Europallet madera } \\
\text { certificada }\end{array}$ & & & & & \\
\hline & CANTIDAD CAJAS & 3.200 & & & & & \\
\hline & $\begin{array}{l}\text { VALOR EXW POR UNIDAD DE } \\
\text { VENTA }\end{array}$ & 1,69 & USD & 22.338 & & & \\
\hline \multirow{5}{*}{$\begin{array}{l}\text { INFORMACION BASICA DEL } \\
\text { EMBARQUE }\end{array}$} & ORIGEN & AEROPUERTO BOGOTÁ - COLO & MBIA & & & & \\
\hline & DESTINO & Puerto Le Havre-Francia & & & & & \\
\hline & PESO TOTAL (KGITON) & 13.200 & & & & & \\
\hline & UNIDAD DE CARGA & PALLETIZADA FCL & & & & & \\
\hline & VOLUMEN TOTAL (CM3/M3) & 20 pallet & & & & & \\
\hline \multirow{4}{*}{ OTRA INFORMACION } & TERMINO DE VENTA & FOB-CIF & & & & & \\
\hline & INFORMACION DE PAGO & & & & & & \\
\hline & TIEMPO DE PAGO & & & & & & \\
\hline & TRM REFERENCIA 02-04-2018 & $3.190,94$ & & & & & \\
\hline \multirow{2}{*}{\multicolumn{3}{|c|}{ CONCEPTOS }} & \multicolumn{5}{|c|}{ UPS } \\
\hline & & & \multicolumn{2}{|c|}{ Costo Unitario } & Costo Total & Tiempo (DIAS) & Observaciones \\
\hline \multirow{16}{*}{$\begin{array}{l}\text { G } \\
\text { A } \\
\text { S } \\
\text { T } \\
0 \\
\text { S } \\
\\
0 \\
\text { R } \\
\text { I } \\
\text { G } \\
\text { E } \\
\text { N }\end{array}$} & & VALOR EXW & USD & 1,69 & 22.308 & & \\
\hline & Elaboración documentos & & USD & 23 & USD & & * documento \\
\hline & Comisión de agente de aduana & & USD & 300.000 & 300.000 & & \\
\hline & Tarifa Ad-Valorem & & 0.35 & & 0.35 & & $\begin{array}{l}\text { Sobre el valor CIF de la } \\
\text { mercancia }\end{array}$ \\
\hline & EMPAQUE & & USD & - & USD & & precio*KG \\
\hline & \begin{tabular}{|l} 
EMBALAJE \\
\end{tabular} & & USD & - & USD & & precio*caja \\
\hline & Certificado de origen & & USD & 10 & USD & & Si aplica \\
\hline & Inland Flete Fresno-Bogotá & & USD & 2.000 & 2.000 & 2 & \\
\hline & Seguro $0,30 \%$ & & USD & 0,00873 & USD & & $\begin{array}{l}\text { Sobre el valor FOB de la } \\
\text { mercancia }\end{array}$ \\
\hline & Combustible & & USD & - & USD & & \\
\hline & Alquiler genset & & USD & - & USD & & *Dia \\
\hline & Certificado INVIMA & & USD & 20 & USD & & $\mathrm{c} / \mathrm{U}$ \\
\hline & Certificado ICA & & USD & 20 & USD & & $\mathrm{c} / \mathrm{U}$ \\
\hline & Gastos operativos y varios & & USD & 50 & USD & & \\
\hline & & VALOR FOB & USD & 302.125 & 324.543 & & \\
\hline & & & \multicolumn{5}{|c|}{ MSC } \\
\hline \multirow{17}{*}{$\begin{array}{l}\text { T } \\
\text { R } \\
\text { A } \\
\text { N } \\
\text { S } \\
\text { I } \\
\text { O } \\
\text { I }\end{array}$} & Transporte Internacional & & USD & 6.000 & 6.000 & 18 & \\
\hline & Equipo de Atmofera controlada & & USD & 1.500 & 1.500 & & \\
\hline & Descarge del contenedor & & USD & 80 & USD & 1 & \\
\hline & Almacenamiento en puerto & & USD & 80 & USD & 3 & \\
\hline & Doc Fee (documentacion) & & USD & 60 & USD & & \\
\hline & \begin{tabular}{|l|} 
Mounting \\
\end{tabular} & & USD & 70 & USD & & ${ }^{*}$ contenedor \\
\hline & Servicio llenado & & USD & 300 & USD & & \\
\hline & Pesaje & & USD & 55 & USD & & \\
\hline & Sellos de seguridad & & USD & 60 & USD & & \\
\hline & Energia*dia & & USD & 80 & USD & 3 & \\
\hline & Coordinaciòn pre inspeccion & & USD & 60 & USD & 1 & \\
\hline & Manejo & & USD & 150 & USD & & *contenedor \\
\hline & Seguro Internacional & & USD & 100 & USD & & \\
\hline & Inspecciones & & USD & 700 & USD & 1 & \\
\hline & & VALOR CIF & USD & 311.420 & 334.158 & 29 & \\
\hline & & & & & & USD $\quad 25,31$ & $\begin{array}{|ll|}\text { USD } & 334.157,69 \\
\end{array}$ \\
\hline & & & & & & POR KILO & TOTAL OPERACIÓN \\
\hline
\end{tabular}

Fuente: Elaboración Propia 
Anexo 5 Costo aéreo por pallet
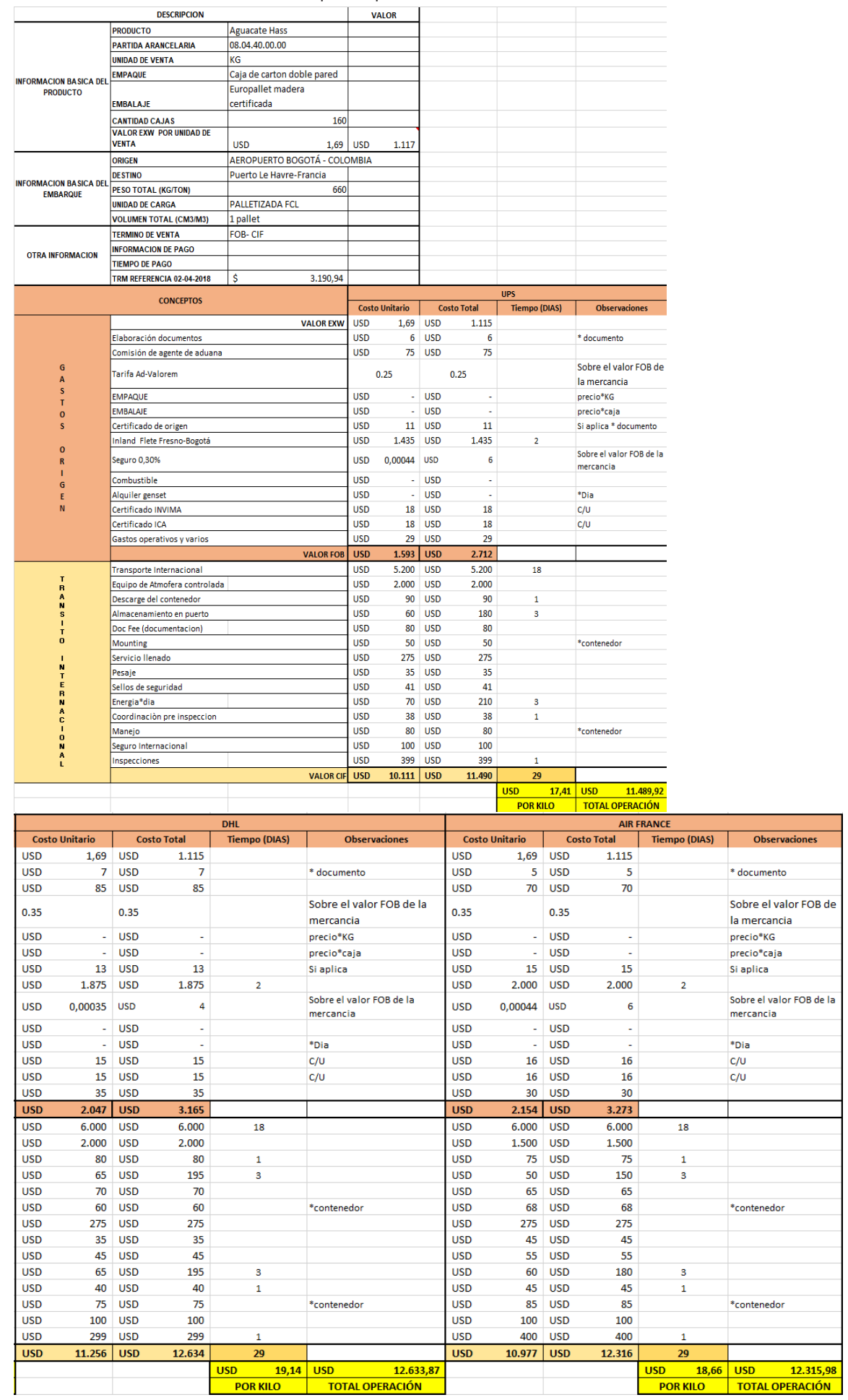

Fuente: Elaboración Propia 